\title{
Digestive System Intraepithelial Neoplasia
}

National Cancer Institute

\section{Source}

National Cancer Institute. Digestive System Intraepithelial Neoplasia. NCI Thesaurus.

Code C8962.

A non-invasive neoplastic process that affects the epithelium of any part of the digestive system. It is characterized by the presence of morphologic features of dysplasia (e.g., architectural and cytological epithelial alterations) or, in a minority of cases, lack of such morphologic features, as in colonic sessile serrated adenomas/polyps. 